\title{
Disordered Gambling: The Effects of a Brief, Online Cognitive Intervention in an Australian Sample
}

\author{
Lucy Jayne Ellison ${ }^{1}$, Kirsten Vale ${ }^{2}$, Robert Ladouceur ${ }^{3}$ \\ ${ }^{1}$ Psychological Science (Honours), Flinders University, Adelaide, South Australia \\ ${ }^{2}$ Flinders University, Adelaide, South Australia \\ ${ }^{3}$ School of Psychology, Université Laval, Québec, Canada \\ Email: *elli0394@flinders.edu.au
}

How to cite this paper: Ellison, L.J., Vale, K. and Ladouceur, R. (2017) Disordered Gambling: The Effects of a Brief, Online Cognitive Intervention in an Australian Sample. Open Access Library Journal, 4: e3288. http://dx.doi.org/10.4236/oalib.1103288

Received: December 9, 2016

Accepted: January 6, 2017

Published: January 9, 2017

Copyright ( 2017 by authors and Open Access Library Inc.

This work is licensed under the Creative Commons Attribution International License (CC BY 4.0).

http://creativecommons.org/licenses/by/4.0/ (c) (i) Open Access

\begin{abstract}
Cognitive processes, in particular, erroneous beliefs surrounding gambling outcomes and abilities, are thought to play an important role in the development and maintenance of disordered gambling behaviours. The current study investigated the impact of erroneous gambling-related cognitions on gambling severity and intentions to gamble, trialling a brief, online cognitive intervention designed to reduce gambling-related cognitions and intentions to gamble. One hundred and twenty one participants were randomly allocated to either an educational intervention condition or an alternative intervention (control condition). Participants in the educational intervention condition showed larger reductions in gambling-related cognitions than did participants in the control condition. The educational intervention significantly reduced erroneous cognitions from pre to post treatment, with effectiveness of the intervention increasing in those exhibiting more severe gambling behaviours.
\end{abstract}

\author{
Subject Areas \\ Psychology \\ Keywords \\ Disordered Gambling, Cognitions, Online Intervention
}

\section{Introduction}

While gambling is a popular and manageable recreational activity for many, it has become problematic for a significant proportion of society, with the majority of prevalence studies reporting rates of disordered gambling between $0.5 \%-2 \%$ 
in the adult population [1] [2]. The Productivity Commission [3] estimated that the average prevalence rate for disordered gambling in Australia was 0.69\%, with a further $1.67 \%$ of the population experiencing moderate to severe negative impacts from gambling such as severe financial, social, physical, psychological, and legal consequences. Furthermore, for each individual with a gambling problem, around 5 to 10 close others are impacted by their gambling; translating to approximately 5 million Australians experiencing adverse consequences associated with gambling [4].

The explanations of why people gamble and the consequences of problem gambling are complex and not yet fully understood. Some argue for dispositional causes while others believe it is fundamentally cognitive or behavioural in nature. Blaszczynski and Nower[5] offered a pathways model which integrates potential influences from a variety of factors including biological, personality, developmental, cognitive, learning, and ecological variants. According to Blaszczynski and Nower [5], gambling beliefs develop as a result of biases in the processing of information in regard to winning and losing (i.e., wins are due to skill and losses to external factors), the role of skill and strategies and the extent to which outcomes can be predicted. Moreover, as gambling escalates and losses continue to accrue, gamblers become trapped into the process of trying to recoup their losses [6]. As such, cognitive processes appear to be fundamental to disordered gambling.

\subsection{Gambling-Related Cognitions}

Cognitive distortions are inherent to disordered gambling, with most gamblers holding inaccurate beliefs about their gambling behaviour and likely outcomes. It is thought that these cognitive distortions both maintain and exacerbate disordered gambling. All gambling activities are games of chance (except poker); however, many gamblers interpret their gambling as a matter of skill, believing they have the capacity to influence the outcome of the game, creating false illusions of control [7] [8]. Such distortions lead to beliefs that gambling wins may be predicted, and result in overconfidence in hope and predictions of winning [8].

In comparison to healthy controls, disordered gamblers have been shown to exhibit higher frequencies of erroneous gambling-related beliefs and hold a greater conviction in these beliefs. Even infrequent gamblers exhibit similar beliefs to disordered gamblers, albeit to a lesser extent [8] [9] [10]. Toneatto, Blitz-Miller, Calderwood, Dragonetti and Tsanos [11] stated that $92 \%$ of disordered gamblers held erroneous gambling-related beliefs. The authors found that disordered gamblers often exhibited behavioural patterns reflecting irrational beliefs such as; talking to Electronic Gaming Machines (EGMs), becoming angry when another patron used a favoured machine, hitting EGMs, as well as making statements indicating that the presence of others influenced their "luck" [12].

\subsection{Cognitive-Behavioural Treatments for Gambling}

As erroneous beliefs have been shown to play a fundamental role in the devel- 
opment and maintenance of gambling behaviours, cognitive approaches in the treatment of disordered gambling aim to challenge erroneous beliefs about gambling outcomes [7] [8] [11]. This is achieved through the examination of individually held beliefs, provision of factual information and restructuring of erroneous automatic thoughts [13]. Cognitive techniques are often combined with behavioural interventions such as financial management, relaxation, and systematic desensitisation, whereby gamblers are gradually exposed to gamblingrelated cues [13]. The combination of behavioural and cognitive techniques has been successful in reducing the frequency of gambling activities and gamblingrelated cognitions in disordered gamblers [14] [15]; although a recent Cochrane review has highlighted the need for further research to measure the long term effects of these findings [16].

\subsection{Cognitive Treatments for Gambling}

Despite the successes of cognitive-behavioural treatment methods, there is evidence to suggest that approaches relying purely on cognitive interventions are also effective in reducing gambling beliefs and frequency of gambling behaviour [17] [18] [19]. For example, Ladouceur et al. [18] evaluated the efficacy of a cognitive treatment model aiming to correct erroneous beliefs around the concept of randomness, the independence of events, and control over gambling outcomes. The results indicated that the majority of the participants displayed increased control over their gambling activities and reduced their urges to gamble at six months follow-up.

This finding was replicated more recently in a randomised-controlled trial in Australia comparing the effectiveness of cognitive therapy with exposure therapy [20]. Participants were 87 individuals with moderate to severe gambling problems, as measured by the Victorian Gambling Screen. Participants were randomly allocated to either the cognitive therapy condition or the exposure therapy condition, and were eligible for up to 12 -60-minute individual treatment sessions, taking place on a weekly basis. Cognitive therapy focused on misconceptions about gambling, such as the concept of randomness, whilst exposure therapy involved extinguishing the urge to gamble through exposure to gambling cues with response elimination. Results indicated that both cognitive therapy and exposure therapy were equally effective in reducing gambling severity symptoms from pre to post-treatment and at a 6-month follow-up. Interestingly, there were fewer drop-outs within the cognitive condition, perhaps due to the relatively confronting nature of exposure therapy.

\subsection{Brief Cognitive Interventions for Disordered Gambling}

Cognitive-behavioural and cognitive treatment methods generally take place over a period of weeks or months. Despite the successes of these methods, there is evidence to suggest that briefer cognitive interventions may be effective in reducing anxiety and fears relating to negative evaluations in social phobia, reducing the frequency and believability of negative cognitions in specific phobias, 
and increasing restraint and ability to manage food cravings [21] [22].

Current literature examining the efficacy of brief cognitive interventions for disordered gambling, although limited, indicates that brief interventions may be helpful in reducing erroneous gambling-related beliefs [23] [24] [25]. Williams and Connolly [25] found that the provision of information explaining probability resulted in increased resistance to gambling fallacies; however, this increased resistance did not translate in reducing gambling behaviour. On the other hand, Floyd, Whelan and Meyers [23] found that individuals who received a brief educational intervention prior to gambling along with brief messages addressing irrational gambling beliefs whilst gambling, reported fewer irrational beliefs and took fewer risks whilst engaging in gambling activities. It is possible that the laboratory setting and presence of a researcher mayreduce the external validity of the findings. Toneatto and Gunaratnee [24] compared a brief cognitive intervention which focused on identifying and restructuring erroneous gambling cognitions, with three brief treatment programs; behavioural therapy, motivational interviewing, and a "minimal intervention" which involved a 90-minute advice-oriented feedback session coupled with the provision of a booklet of practical strategies. All four treatment programs were equally effective in reducing gambling frequency and symptom severity, with significant improvements through to twelve months post treatment [24]. This approach is important to consider in terms of the affordability and accessibility of treatment as well as managing waiting lists. Given that the current literature reflects outcomes with clinical populations, it is also proposed that brief programs may be successfully applied as preventative strategies with at-risk groups.

Whilst there is little evidence on the optimal mode of delivery for gambling interventions, Phillips and Blaszczynski [26] suggested that online treatments for disordered gambling are likely to foster increased participation due to their convenience, accessibility, privacy and cost-effectiveness. Online interventions for disordered gamblers are a relatively new treatment option and there is a lack of empirical evidence surrounding their effectiveness [27]. Despite this, there is a significant amount of research supporting the effectiveness of online interventions for other addictions including alcohol abuse and smoking [28] [29] [30]. Comparisons with face-to-face therapy revealed that online interventions for addictions are equally effective in improving client knowledge and achieving positive behavioural and clinical outcomes [27] [31]. It is clear that online interventions have an important role in the treatment of addictions; which suggests that further research regarding the efficacy of online interventions specifically designed for disordered gamblers, may be beneficial. Research by Toneatto et al. [32] indicates that individuals with mild to moderate gambling problems do not necessarily require formal interventions, suggesting that the development of online interventions may be particularly relevant to this population.

Given the extent to which erroneous cognitions have been shown to maintain the cycle of disordered gambling and the early evidence suggesting that brief interventions may be helpful, the current study investigates the impact of a brief, 
online educational intervention designed to reduce erroneous gambling beliefs in a non-clinical sample.

\section{Method}

\subsection{Participants}

One hundred and twenty one Australian undergraduate psychology students (27 males and 94 females) aged between 17 and 69 years old $(M=24.79, S D=11.16)$ were recruited to take part in an online study assessing gambling habits. As expected, mean scores for gambling severity $(M=5.01, S D=7.94)$ were below the cut-off score which clinically defines disordered gambling on the Victorian Gambling Screen (VGS), indicating that the sample of participants were not a population with severe gambling problems. Participants reported low frequencies of gambling behaviour, with the majority of participants indicating that they had not gambled within the last month. Participants who did engage in gambling activities reported participation in a range of activities including electronic gaming machines, sports and horse betting, lotteries, card/table games and raffle tickets.

\subsection{Procedure}

Participants answered basic demographic questions, provided information about their gambling behaviour in the last four weeks, and indicated their current intentions to gamble. Participants were then asked to complete the Gambling Related Cognitions Scale (GRCS) [33] and Victorian Gambling Screen (VGS) [34]. Following completion of the questionnaires, participants were randomly allocated to either an educational intervention or the alternative intervention. Both interventions required participants to read a short text about gambling. Participants were not aware that the content of these texts differed. All participants, regardless of which intervention they received, were asked to complete the GRCS for a second time, and to report their intentions to gamble. This process took approximately 30 minutes to complete.

\subsection{Materials}

Victorian Gambling Screen (VGS). The VGS [34], is a self-report questionnaire containing 21 items and three subscales; harm-to-self, harm-to-others, and enjoyment of gambling. Despite the usefulness of the latter two scales, validation studies indicate they do not meaningfully identify disordered gamblers; therefore, the 15-item harm-to-self scale ( $\alpha=0.89)$ was used in this study [13] [35]. Items are scored on a five point Likert scale ranging from 0 (never) to 4 (always). Scores can range from $0-60$, with scores of 21 or above indicating disordered gambling.

Gambling Related Cognitions Scale (GRCS). The GRCS [33] is a self-report questionnaire designed to assess erroneous thoughts about gambling, and demonstrates good internal consistency $(\alpha=0.93)$ in non-clinical populations. The GRCS contains 23 items whereby participants indicate the extent to which they 
agree with each statement on a seven-point Likert scale ranging from 0 (strongly disagree) to 6 (strongly agree) with higher scores indicating more erroneous beliefs about gambling.

Educational Intervention. The educational intervention involved participants reading a short text about gambling. The intervention was designed to target erroneous beliefs about gambling and included information specifically challenging beliefs about chance, luck and control. The text was taken from a disordered gambling treatment manual [8]. The text was modified slightly in order to increase relevance to an Australian population.

Alternative Intervention. The alternative intervention also involved participants reading a short passage about gambling. The passage provided informative factual information about gambling prevalence and types in Australia. This passage was designed to be interesting to readers, yet was worded such that the information was neutral and did not provide support for either pro or anti-gambling perspectives. The information for this intervention was drawn from a gambling report publicly available on the website of the Australian Psychological Society [36].

\section{Results}

Mean responses and possible range of scores for all variables are presented in Table 1. As expected for a non-clinical group, mean scores for gambling severity were well below the cut-off score which clinically defines disordered gambling on the VGS. Measures of gambling related cognitions indicated that participants did not experience high levels of irrational beliefs relating to their gambling behaviours and outcomes. Intentions to gamble amongst the sample were also relatively low.

Table 1. Mean scores and standard deviations.

\begin{tabular}{cccc}
\hline Variable & Range & $\mathrm{N}$ & Mean (SD) \\
\hline Gambling Severity & $0-60$ & 121 & $5.01(7.94)$ \\
Cognitions (Before) & & 121 & $17.74(18.27)$ \\
Educational Intervention & $0-138$ & 71 & $18.01(18.79)$ \\
Alternative Intervention & & 50 & $17.36(17.68)$ \\
Cognitions (After) & $0-138$ & 71 & $11.01(16.04)$ \\
Educational Intervention & & 50 & $9.62(14.84)$ \\
Alternative Intervention & & $12.98(17.58)$ \\
Intention (Before) & & 121 & $1.16(1.18)$ \\
Educational Intervention & & 71 & $1.17(1.15)$ \\
Alternative Intervention & & 50 & $1.08(1.19)$ \\
Intention (After) & & 121 & $1.02(1.24)$ \\
Educational Intervention & & 50 & $1.04(1.27)$ \\
Alternative Intervention & & & \\
\hline
\end{tabular}




\subsection{Gambling-Related Cognitions as a Predictor of Intentions to Gamble and Gambling Severity}

Two bivariate linear regression analyses were conducted in order to determine whether gambling severity and intentions to gamble could be predicted from gambling-related cognitions. Gambling-related cognitions predicted intentions to gamble, $\mathrm{R}^{2}=0.26, F(1,119)=43.08, p<0.001$, and gambling severity, $\mathrm{R}^{2}=$ $0.39, F(1,119)=75.99, p<0.001$.

\subsection{The Impacts of an Educational Cognitive Intervention on Gambling-Related Cognitions and Intentions to Gamble}

Two independent samples t-tests were conducted to compare the change in cognitions and intentions to gamble between the educational and alternative interventions. The education group had significantly fewer gambling-related cognitions $(M=-8.39, S D=13.54)$ than the group receiving the alternative intervention $(M=-4.38, S D=8.28) ; t(119)=2.02, p=0.046$, two-tailed, Cohens $d=0.3$. There was no significant differences between the educational $(M=-0.04, S D=$ $0.15)$ and alternative $(M=-0.03, S D=0.09)$ interventions in terms of intentions to gamble; $t(119)=-0.52, p=0.607$, two-tailed.

A hierarchical multiple regression was conducted to determine whether gambling severity moderated the relationship between the educational intervention and change in gambling-related cognitions. The educational intervention was more effective in reducing gambling-related cognitions in those with more severe gambling behaviours. Individuals displaying more severe gambling behaviours were more likely to benefit from the educational intervention. Regression equations can be found in Table 2 .

An interaction plot for intervention, gambling severity and change in cognitions can be found in Figure 1. Examination of the interaction plot indicated that the educational intervention was more effective in reducing gambling-related cognitions than was the alternative intervention, and that the effectiveness of the educational intervention in reducing these beliefs increased as gambling severity increased. Mean change in cognition scores for the alternative intervention remained similar regardless of the level of gambling severity.

Table 2. Multiple regression assessing the effects of gambling severity on the impacts of an educational intervention designed to reduce gambling-related cognitions.

\begin{tabular}{cccccc}
\hline & Adjusted $\mathrm{R}^{2}$ & $\mathrm{R}^{2}$ Change & F Change & $\beta$ & $\mathrm{t}$ \\
\hline $\begin{array}{c}\text { Gambling-related Cognitions } \\
\text { Step 1 }\end{array}$ & 0.020 & 0.028 & 30.474 & & \\
$\begin{array}{c}\text { Intervention } \\
\text { Step 2 }\end{array}$ & & & & 0.168 & 10.864 \\
Gambling Severity & 0.077 & 0.064 & $80.343^{* *}$ & & \\
$\quad$ Step 3 & & & & -0.253 & $-20.888^{* *}$ \\
Intervention ${ }^{*}$ Gambling Severity & 0.114 & 0.044 & $50.935^{*}$ & & \\
\hline${ }^{*} p<0.05, p<0.01,{ }^{* *} p<0.001$ (2-tailed). & & & 0.642 & $20.436^{*}$ \\
\hline
\end{tabular}




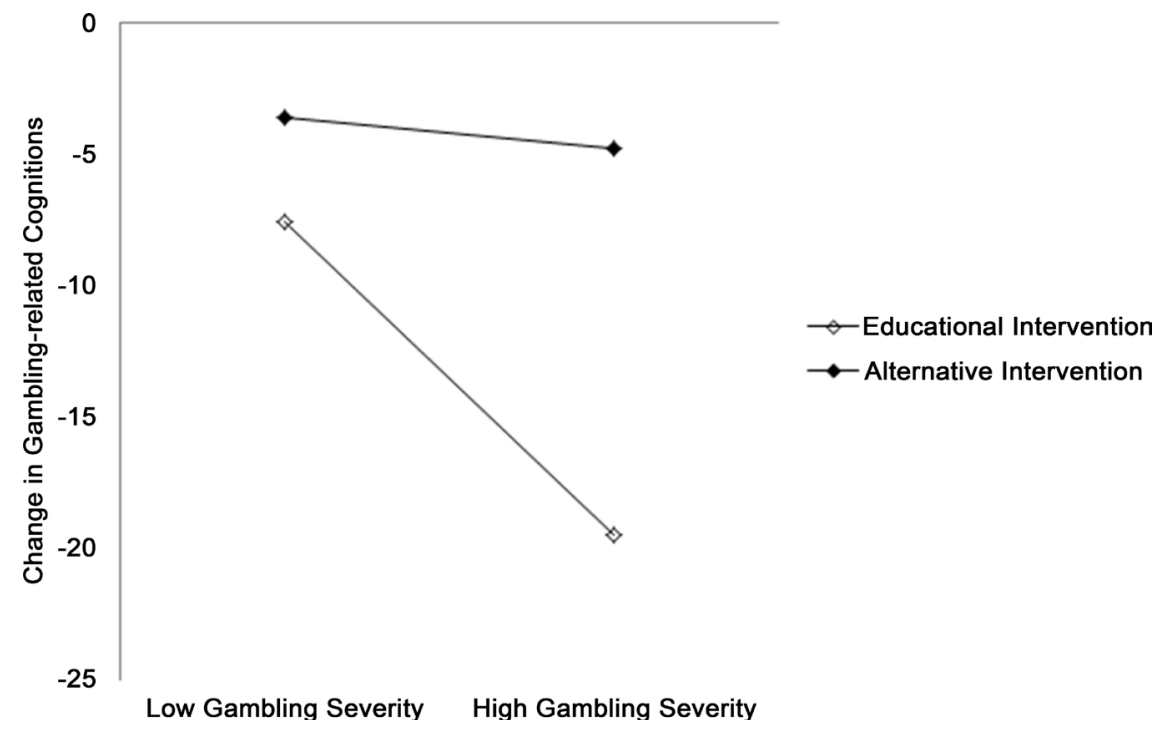

Figure 1. Interaction plot for intervention, gambling severity and change in cognitions.

\section{Discussion}

The present study investigated the impact of a brief cognitive intervention designed to challenge gambling-related cognitions and intentions to gamble. The study also investigated whether gambling severity impacted the effectiveness of the intervention in reducing gambling-related cognitions and intentions to gamble. Whilst the educational intervention was found to reduce erroneous gambling-related cognitions, it did not influence intentions to gamble. In terms of clinical implications, individuals with high levels of gambling severity experienced larger reductions in gambling-related cognitions than did individuals with lower levels of gambling severity.

Previous results have identified specific unhelpful cognitions and thought processes which underlie dysfunctional gambling behaviours, particularly those relating to luck, chance and skill [10] [13]. The role of these cognitions in maintaining and exacerbating gambling behaviours has been established in both clinical and non-clinical samples, with higher frequencies of gambling-related cognitions and a greater conviction in these cognitions resulting in more severe gambling behaviours [13] [37]. Based on these findings, it is not surprising that the current study also found links between gambling-related cognitions and gambling severity, and gambling-related cognitions and intentions to gamble. Although participants were not from a clinical population, gambling-related cognitions explained a significant proportion of the variance in gambling severity and gambling intentions, with large effect sizes demonstrating the important role of unhelpful cognitions in the development of gambling behaviours in a non-clinical population. These findings support previous research in establishing a strong association between gambling-related cognitions and severity of gambling behaviours. This result provides a promising foundation for the development of efficacious treatment methods for disordered gambling and suggests that treatment programs aimed at reducing erroneous gambling-related 
cognitions may be effective in reducing both severity of gambling behaviours and intentions to engage in harmful gambling behaviours [24].

In treatment settings, cognitive treatments that involve the provision of information aimed to dispute erroneous cognitions, have demonstrated effectiveness in reducing gambling behaviours and intentions to gamble [18]. Comparisons with cognitive-behavioural treatment methods indicate that purely cognitive treatments are equally as effective as combined cognitive-behavioural techniques in reducing gambling behaviours and intentions to gamble, which can be explained by the significant role of erroneous cognitions in the development of disordered gambling behaviours [18] [24]. However, despite the fundamental role of cognitions in disordered gambling, previous empirical research investigating the effectiveness of cognitive treatments for gambling has failed to include measures of gambling-related cognitions, making it difficult to assess whether there were changes in cognitions from pre to post treatment. Furthermore, little has been done to evaluate the effectiveness of brief cognitive interventions, nor the impact of these administered online.

Although previous research examining the efficacy of brief cognitive interventions for disordered gambling is limited, the present study demonstrated that the information provided in a brief cognitive intervention modified erroneous gambling beliefs, at least in the short-term, and provides useful insight for clinicians developing treatment programs for disordered gambling. Whilst the intervention was primarily designed to reduce gambling-related cognitions, it was also hypothesized that a reduction in erroneous cognitions would reduce the intentions to gamble. Unexpectedly, this was not the case; intentions to gamble remained constant from pre to post intervention. Whilst this is likely due to low baseline frequencies for intentions to gamble in the sample, making significant reductions in intentions to gamble unlikely; Steenbergh, Whelan, Meyers, May, \& Floyd [38] suggested that changes in knowledge do not necessarily translate to changes in behaviour.

The reductions in erroneous gambling-related cognitions are a promising indication of the effectiveness of brief online interventions; however, without a measure of gambling-related cognitions at a follow-up after the intervention, it is difficult to determine the length of the effects. Future research incorporating these measures would enable clinicians to determine how such an intervention may be utilised in the treatment of disordered gambling. Although it is unlikely that such a brief intervention would be adequate as the sole form of treatment for disordered gambling. Providing information to clients who are on waiting lists for treatment or attaching this information to community websites may be a helpful tool to those who are unsure about seeking professional help or those who cannot afford treatment. Given that the intervention was more effective in those with more severe gambling problems, it would also be beneficial to further explore the impacts of a brief cognitive intervention in a clinical sample. This may yield larger effect sizes than studies using non-clinical populations, and also give an indication as to whether the intervention has any success in reducing in- 
tentions to gamble in a population classified as disordered gamblers, whose intentions to gamble are anticipated to be more dominant than in the current sample. Given the strong association between gambling-related cognitions and gambling behaviours, the findings of this study also have clinical implications in terms of the prevention of disordered gambling. Increasing knowledge and awareness around common gambling misconceptions and challenging erroneous gambling-related cognitions may be useful in preventing disordered gambling behaviours [39].

\section{Conclusion}

In conclusion, the present study demonstrates the immediate benefits of a brief cognitive intervention and provides a foundation for future research surrounding the development of prevention methods and online cognitive treatments for disordered gambling.

\section{References}

[1] Australian Institute of Health and Welfare (2006) Australia's Health 2006. http://www.aihw.gov.au/WorkArea/DownloadAsset.aspx?id=6442453483

[2] Delfabbro, P. and King, D. (2012) Gambling in Australia: Experiences, Problems, Research and Policy. Addiction, 107, 1556-1561. https://doi.org/10.1111/j.1360-0443.2012.03802.x

[3] Productivity Commission (2010) Gambling: Productivity Commission Inquiry Report.

http://www.pc.gov.au/_data/assets/pdf_file/0010/95680/gambling-report-volume1.pdf

[4] Australian Medical Association (2013) Health Effects of Disordered Gambling. http://ama.com.au/position-statement/health-effects-problem-gambling

[5] Blaszczynski, A. and Nower, L. (2002) A Pathways Model of Problem and Pathological Gambling. Addictions, 97, 487-499. https://doi.org/10.1046/j.1360-0443.2002.00015.x

[6] Walker, M.B. (1992) Irrational Thinking among Slot Machine Players. Journal of Gambling Studies, 8, 245-261. https://doi.org/10.1007/BF01014652

[7] Clark, L. (2010) Decision-Making during Gambling: An Integration of Cognitive and Psychobiological Approaches. Philosophical Transactions of the Royal Society of Biological Sciences, 365, 319-330. https://doi.org/10.1098/rstb.2009.0147

[8] Ladouceur, R. and Lachance, S. (2007) Overcoming Pathological Gambling: Therapist Guide. Oxford University Press, New York.

[9] Griffiths, M.D. (1994) The Role of Cognitive Bias and Skill in Fruit Machine Gambling. British Journal of Psychology, 85, 351-369. https://doi.org/10.1111/j.2044-8295.1994.tb02529.x

[10] Michalzuk, R., Bowden-Jones, H., Verdejo-Garcia, A. and Clark, L. (2011) Impulsivity and Cognitive Distortions in Pathological Gamblers Attending the UK National Problem Gambling Clinic: A Preliminary Report. Psychological Medicine, 41, 2625-2635. https://doi.org/10.1017/S003329171100095X

[11] Toneatto, T., Blitz-Miller, T., Calderwood, K., Dragonetti, R. and Tsanos, A. (1997) Cognitive Distortions in Heavy Gambling. Journal of Gambling Studies, 13, 253 266. https://doi.org/10.1023/A:1024983300428

[12] Delfabbro, P., Osborn, A., Nevile, M., Skelt, L. and McMillen, J. (2007) Identifying 
Problem Gamblers in Gambling Venues: Final Report. Gambling Research Australia, Melbourne.

[13] Delfabbro, P. (2011) Australasian Gambling Review: 5th Edition. http://iga.sa.gov.au/sites/default/files/publication-documents/Australian\%20Gambli ng\%20Review-5th\%20Edition.pdf

[14] Dowling, N., Smith, D. and Thomas, T. (2006) Treatment of Female Pathological Gambling: The Efficacy of a Cognitive Behavioural Approach. Journal of Gambling Studies, 22, 355-372. https://doi.org/10.1007/s10899-006-9027-3

[15] Oei, T.P.S., Raylu, N. and Casey, L.M. (2010) Effectiveness of Group and Individual Formats of a Combined Motivational Interviewing and Cognitive Behavioural Treatment Program for Problem Gambling: A Randomised Controlled Trial. Behavioural and Cognitive Psychotherapy, 38, 233-238. https://doi.org/10.1017/S1352465809990701

[16] Cowlishaw, S., Merkouris, S., Dowling, N., Anderson, C., Jackson, A. C. and Thomas, S. (2012) Psychological Therapies for Pathological and Problem Gambling. Cochrane Database of Systematic Reviews, 11, CD008937. https://doi.org/10.1002/14651858.CD008937.pub2

[17] Echeburua, E., Baez, C. and Fernandez-Montalvo, J. (1996) Comparative Effectiveness of Three Therapeutic Modalities in the Psychological Treatment of Pathological Gamblers: Long-Term Outcome. Behavioural and Cognitive Psychotherapy, 24, 51-72. https://doi.org/10.1017/S1352465800016830

[18] Ladouceur, R., Sylvain, C., Letarte, H., Giroux, I. and Jacques, C. (1998) Cognitive Treatment of Pathological Gamblers. Behaviour Research and Therapy, 36, 11111119. https://doi.org/10.1016/S0005-7967(98)00086-2

[19] Sylvain, C., Ladouceur, R. and Boisvert, J.M. (1997) Cognitive and Behavioural Treatment of Pathological Gambling: A Controlled Study. Journal of Consulting and Clinical Psychology, 65, 727-732. https://doi.org/10.1037/0022-006X.65.5.727

[20] Battersby, M., Smith, D., Harvey, P. and Pols, R. (2013) Cognitive versus Exposure Therapy for Problem Gambling: A Pilot Randomised Controlled Trial. Victorian Responsible Gambling Foundation, Victoria.

[21] Clark, D.M., Ehlers, A., McManus, F., Hackmann, A., Fennell, M., Campbell, H., Louis, B., et al. (2003) Cognitive Therapy versus Fluoxetine Therapy in Generalized Social Phobia: A Randomized Placebo-Controlled Trial. Journal of Consulting and Clinical Psychology, 71, 1058-1067. https://doi.org/10.1037/0022-006X.71.6.1058

[22] Moffitt, R., Brinkworth, G., Noakes, M. and Mohr, P. (2012) A Comparison of Cognitive Restructuring and Cognitive Defusion as Strategies for Resisting a Craved Food. Journal of Psychology and Health, 27, 74-90. https://doi.org/10.1080/08870446.2012.694436

[23] Floyd, K., Whelan, J. P. and Meyers, A. (2006) Use of Warning Messages to Modify Gambling Beliefs and Behaviour in a Laboratory Investigation. Psychology of Addictive Behaviours, 20, 69-74. https://doi.org/10.1037/0893-164X.20.1.69

[24] Toneatto, T. and Gunaratne, M. (2009) Does the Treatment of Cognitive Distortions Improve Clinical Outcomes for Disordered Gambling? Journal of Contemporary Psychotherapy, 39, 221-229. https://doi.org/10.1007/s10879-009-9119-3

[25] Williams, R.J. and Connolly, D. (2006) Does Learning about the Mathematics of Gambling Change Gambling Behaviour? Psychology of Addictive Behaviours, 20, 62-68. https://doi.org/10.1037/0893-164X.20.1.62

[26] Phillips, J.G. and Blaszczynski, A. (2010) Gambling and the Impact of New and Emerging Technologies and Associated Products. 
https://www.gamblingresearch.org.au/sites/default/files/embridge_cache/emshare/origina 1/public/2016/09/96/375e3e9af/gra\%2Bemerging\%2Btechnologies\%2Bfinal\%2Breport.pdf

[27] Gainsbury, S. and Blaszczynski, A. (2011) Online Self-Guided Interventions for the Treatment of Problem Gambling. International Gambling Studies, 11, 289-308. https://doi.org/10.1080/14459795.2011.617764

[28] Etter, J.F. (2005) Comparing the Efficacy of Two Internet-Based, Computer-Tailored Smoking Cessation Programs: A Randomized Trial. Journal of Medical Internet Research, 7, e2. https://doi.org/10.2196/jmir.7.1.e2

[29] Severson, H.H., Gordon, J.S., Danaher, B.G. and Akers, L. (2008) ChewFree.com: Evaluation of a Web-Based Cessation Program for Smokeless Tobacco Users. Nicotine\& Tobacco Research, 10, 381-391. https://doi.org/10.1080/14622200701824984

[30] White, A., Kavanagh, D., Stallman, H., Klein, B., Kay-Lambkin, F., Proudfood, J., Young, R., et al. (2010) Online Alcohol Interventions: A Systematic Review. Journal of Medical Internet Research, 12, e62. https://doi.org/10.2196/jmir.1479

[31] Murray, E., Burns, J., See, T. S., Lai, R. and Nazareth, I. (2004) Interactive Health Communication Applications for People with Chronic Disease. Cochrane Database of Systematic Reviews, No. 4, CD004274.

[32] Toneatto, T., Cunningham, J., Hodgins, D., Adams, M., Turner, N. and KoskiJannes, A. (2008) Recovery from Problem Gambling without Formal Treatment. Addiction Research and Theory, 16, 111-120. https://doi.org/10.1080/16066350801923638

[33] Raylu, N. and Oei, T.P.S. (2004) The Gambling Related Cognitions Scale (GRCS): Development, Confirmatory Factor Validation and Psychometric Properties. Addiction, 99, 757-769. https://doi.org/10.1111/j.1360-0443.2004.00753.x

[34] Ben-Tovim, D., Esterman, A., Tolchard, B. and Battersby, M.W. (2001) The Victorian Gambling Screen: Project Report. Victorian Research Panel, Melbourne.

[35] Tolchard, B. and Battersby, M.W. (2010) The Victorian Gambling Screen: Reliability and Validation in a Clinical Population. Journal of Gambling Studies, 26, 623 638. https://doi.org/10.1007/s10899-009-9172-6

[36] Australian Psychological Society (2010) The Psychology of Gambling, In Psych: The Bulletin of the Australian Psychological Society, 32, 12-13.

[37] Mobini, S., Pearce, M., Grant, A., Mills, J. and Yeomans, M.R. (2006) The Relationship between Cognitive Distortions, Impulsivity and Sensation Seeking in a NonClinical Population Sample. Personality and Individual Differences, 40, 1153-1163. https://doi.org/10.1016/j.paid.2005.11.006

[38] Steenbergh, T., Whelan, J. A., Meyers, A., May, R. and Floyd, K. (2004) Impact of Warning and Brief Intervention Messages on Knowledge of Gambling Risk, Irrational Beliefs and Behaviour. Journal of International Gambling Studies, 4, 3-16. https://doi.org/10.1080/1445979042000224377

[39] Ladouceur, R., Ferland, F. and Vitaro, F. (2004) Prevention of Problem Gambling: Modifying Misconceptions and Increasing Knowledge among Canadian Youths. Journal of Primary Prevention, 25, 329-335. https://doi.org/10.1023/B:JOPP.0000048024.37066.32 
Submit or recommend next manuscript to OALib Journal and we will provide best service for you:

- Publication frequency: Monthly

- 9 subject areas of science, technology and medicine

- Fair and rigorous peer-review system

- Fast publication process

- Article promotion in various social networking sites (LinkedIn, Facebook, Twitter, etc.)

- Maximum dissemination of your research work

Submit Your Paper Online: Click Here to Submit

Or Contact service@oalib.com 